Draft version May 6, 2019

Preprint typeset using IATEX style emulateapj v. 11/12/01

\title{
MAGNETIC FIELD STRUCTURE IN THE FLATTENED ENVELOPE AND JET IN THE YOUNG PROTOSTELLAR SYSTEM HH 211
}

\author{
Chin-Fei lee ${ }^{1}$, Ramprasad Rao ${ }^{1}$, Tao-Chung Ching ${ }^{2}$, Shih-Ping Lai ${ }^{2}$, Naomi Hirano ${ }^{1}$, Paul \\ T.P. $\mathrm{HO}^{1,3}$, AND HSIANG-CHIH HWANG ${ }^{1}$ \\ Draft version May 6, 2019
}

\begin{abstract}
HH 211 is a young Class 0 protostellar system, with a flattened envelope, a possible rotating disk, and a collimated jet. We have mapped it with the Submillimeter Array in $341.6 \mathrm{GHz}$ continuum and $\mathrm{SiO}$ $\mathrm{J}=8-7$ at $\sim 0.0^{\prime \prime} 6$ resolution. The continuum traces the thermal dust emission in the flattened envelope and the possible disk. Linear polarization is detected in the continuum in the flattened envelope. The field lines implied from the polarization have different orientations, but they are not incompatible with current gravitational collapse models, which predict different orientation depending on the region/distance. Also, we might have detected for the first time polarized $\mathrm{SiO}$ line emission in the jet due to the GoldreichKylafis effect. Observations at higher sensitivity are needed to determine the field morphology in the jet.
\end{abstract}
Subject headings: stars: formation — ISM: individual objects (HH 211) — ISM: magnetic fields - polarization

\section{INTRODUCTION}

Magnetic field has important effects in low-mass star formation. It can launch a collimated jet but may suppress the formation of a rotationally supported (accretion) disk (RSD) in the early phase of star formation. In current theory, gravitational collapse of a rotating magnetized cloud core produces a flattened envelope and an hourglass field morphology around a central source (i.e., protostar) (see, e.g., Allen. Li. \& Shu 2003). The flattened envelope is not rotationally supported and is thus called pseudodisk. In the flattened envelope, rotation velocity increases toward the center, generating a toroidal field in the inner part (Allen, Li, \& Shu 2003; Kataoka et al. 2012). However, the pinched geometry of the magnetic field in the flattened envelope generates a magnetic braking, suppressing the formation of a RSD around the center Allen. Li. \& Shu 2003). One possible solution is to have a misalignment between rotation axis and magnetic field axis (Joos et al. 2012; Li et al. 2013).

Observations of linearly polarized thermal dust emission toward low-mass Class 0 systems have revealed magnetic field morphologies of the envelope in support of the above theoretical picture. They have revealed an hourglass field morphology in, e.g., NGC 1333 IRAS 4A (Girart et al. 2006) and L 1157 (Stephens et al. 2013). They have also showed a tentative detection of toroidal fields near the center in, e.g., NGC 1333 IRAS 4A (Crutcher 2012) and IRAS 16293-2422 B (Rao et al.|2014). Moreover, misalignment between rotation axis and magnetic field axis could be common, as suggested in Hull et al. (2013). However, further observations are still needed to confirm the field morphology near the center and the misalignment.

On the other hand, the field morphology in protostellar jet is poorly determined due to a lack of polarization de- tection. In current jet-launching models (Shu et al. 2000; Pudritz et al. 2007), poloidal field is needed to launch the jet and toroidal field is needed to collimate the jet. Therefore, the jet is expected to be magnetized with a helical field. For high-mass systems, the jet can emit synchrotron radiation, allowing us to map the field morphology with polarization observation in synchrotron continuum (Carrasco-González et al. 2010). For low-mass systems, the jet can emit molecular line emission (Lee et al. 2007; Hirano et al. 2010). Line polarization has been detected in molecular outflows in low-mass systems, e.g., NGC 1333 IRAS 4A (Girart et al. 1999) and high-mass systems, e.g., DR 21 (Lai et al. 2003). It is attributed to the Goldreich-Kylafis (GK) effect (Goldreich \& Kylafis 1981, 1982), and thus can be used to infer the field morphology in the molecular outflows. It can also be used to infer the field morphology in the jet.

HH 211 is a nearby (280 pc) low-mass system, in which a flattened envelope has been detected, a RSD has been claimed, and a collimated jet has been detected in $\mathrm{SiO}$ and $\mathrm{CO}$ down to the launching point inside an outflow cavity (Gueth et al. 1999; Lee et al. 2009, hereafter Lee09). It is young in the Class 0 phase, with significant material still in the flattened envelope and a mass of only $\sim 0.06 M_{\odot}$ for the central source. The jet is almost in the plane of the sky and the flattened envelope is almost edge-on, providing the best view for the fields. Here, we report detections of thermal dust polarization in the flattened envelope and line polarization in the jet, and discuss possible field morphologies in this system.

\section{OBSERVATIONS}

Polarization observation toward HH 211 was carried out with the Submillimeter Array (SMA) (Ho et al. 2004) on 2013 November 28 in the extended configuration, using a

1 Academia Sinica Institute of Astronomy and Astrophysics, P.O. Box 23-141, Taipei 106, Taiwan; cflee@asiaa.sinica.edu.tw

2 Institute of Astronomy and Department of Physics, National Tsing Hua University, Hsinchu, Taiwan

${ }^{3}$ Harvard-Smithsonian Center for Astrophysics, 60 Garden Street, Cambridge, MA 02138

4 The Submillimeter Array is a joint project between the Smithsonian Astrophysical Observatory and the Academia Sinica Institute of Astronomy and Astrophysics, and is funded by the Smithsonian Institution and the Academia Sinica 
dual-receiver mode with the $345 \mathrm{GHz}$ and $400 \mathrm{GHz}$ receivers. $\mathrm{SiO}(J=8-7), \mathrm{CO}(J=3-2)$, and $\mathrm{SO}$ $\left(N_{J}=8_{9}-7_{8}\right)$ lines were observed simultaneously with continuum. Here, we only present the results in continuum and $\mathrm{SiO}$ that show polarization detection. The receivers have two sidebands, lower and upper, covering the frequency range from 335.6 to $337.6 \mathrm{GHz}$ and from 345.6 to $347.6 \mathrm{GHz}$, respectively. Combining the line-free portions of the two sidebands results in a total bandwidth of $\sim$ $3.7 \mathrm{GHz}$ centered at $\sim 341.6 \mathrm{GHz}$ for the continuum. The baselines of the array have projected lengths ranging from $\sim 27$ to $224 \mathrm{~m}$. The primary beam has a size of $\sim 35^{\prime \prime}$. The correlator was set up to have a velocity resolution of $\sim 1.4 \mathrm{~km} \mathrm{~s}^{-1}$.

Quasar 3c84 was used as a gain, a bandpass, and a polarization calibrator. Its fractional linear polarization was determined to be $\sim 0.1 \%$, which is the limit of detectability with the SMA. The absolute flux scale was determined by using the 3c84 flux (6.3 Jy) in the SMA database, with a flux uncertainty of $\lesssim 15 \%$. The visibility data were reduced, with the bandpass and gain calibrations done with the MIR package, and the polarization and flux calibration done with the MIRIAD package. The antenna leakages were found to be up to $\sim 3 \%$ except for antenna 7 which is $\sim 7 \%$. The calibrated visibility data were imaged with the MIRIAD package. With natural weighting, the synthesized beam has a size of $00^{\prime \prime} 70 \times 00^{\prime \prime} 51$ at a position angle (P.A.) of $\sim-88^{\circ}$. The rms noise levels in different maps are given later in figure captions.

\section{FLATTENED ENVELOPE}

At $341.6 \mathrm{GHz}$, the continuum map shows a flattened structure within a radius of $\sim 1^{\prime \prime}$ of the central source, roughly perpendicular to the jet axis (see Fig. 1). The source is assumed to be at the peak position of the continuum, which is $\alpha_{(2000)}=03^{\mathrm{h}} 43^{\mathrm{m}} 56.806, \delta_{(2000)}=$ $32^{\circ} 00^{\prime} 50^{\prime \prime} \cdot 21$, consistent with the position found at higher resolution (Lee09). The jet is known to be bended and wiggling, with a mean axis having a P.A. of $114^{\circ} \pm 4^{\circ}$ (Gueth \& Guilloteau 1999; Lee et al. 2007; Lee09). The continuum has a total flux density of $220 \pm 30 \mathrm{mJy}$, consistent with the value found before. The center is unresolved with a peak flux density of $112 \mathrm{mJy}_{\text {beam }}{ }^{-1}$. A fit to the visibility amplitude versus $u v$-distance plot of the continuum requires two circular Gaussian components; an extended component with a size of $\sim 1$." 8 and a compact component with a size of $\sim 0^{\prime \prime} 3$. Thus, as discussed before, the continuum traces the thermal dust emission from a flattened envelope and a possible compact disk around the source (Lee et al. 2007; Lee09). In Lee09, a faint secondary source was detected at $\sim 00^{\prime \prime} 3$ to the southwest of the central source at higher resolution. However, this source can not be resolved here. The envelope is slightly asymmetric, extending further to the SW than to the NE (probably partly because of the secondary source) and further to the SE than to the NW.

\subsection{Dust Polarization and Field Morphology}

Linear polarization is detected in the thermal dust emission in the SE, NE, SW, and NW parts of the envelope, as shown by the polarization intensity map and vectors in Figure 1 1 . The angles of the polarization vectors have an uncertainty of $\sim 13^{\circ}$. The degree of polarization reaches $\sim 30 \%$ at the edges of the envelope, as found in other systems e.g., NGC 1333 IRAS 4A (Girart et al. 2006). In the SE part, the detection of the polarized emission has a peak of more than $3 \sigma$ and is thus real. In other parts, the detections are 2 to $3 \sigma$ and thus should be considered as tentative.

Figure 1 $\mathrm{b}$ shows the magnetic field lines in the envelope obtained by rotating the polarization vectors by $90^{\circ}$. The field morphology is complicated because the field lines have different orientations in different regions. Along the major axis, the SW field lines are roughly aligned with the jet axis slightly pinched toward the source. However, the NE field lines, which are closer to the central source than the SW field lines, are almost perpendicular to the jet axis. Along the minor axis, the NW field lines appear to be roughly perpendicular to the jet axis. However, the SE field lines, which are further away from the central source than the NW field lines, appear to be roughly aligned with the jet axis.

\subsection{Comparison and Discussion}

As mentioned earlier, current polarization observations support a gravitational collapse model of a rotating magnetized cloud core for low-mass star formation. For the first comparison, we compare to a simple version of such model, in which the field lines are initially poloidal with the field axis aligned with the rotation axis. Because of the gravitational collapse, the field lines are pinched toward the source, forming an hourglass field morphology. Moreover, toroidal fields are generated by rotation in the flattened envelope near the source around the rotation axis. Figure 1r shows the 3D view toward the inner part of such a model from Kataoka et al. (2012), where the toroidal field lines have formed. The model has a spatial scale about 4 times larger because it is 5-10 times older than $\mathrm{HH} 211$, and is thus only used as an illustration of the complex structure of the magnetic field in the envelope. The model has an edge-on view, appropriate for HH 211. It is rotated with the field axis (black lines) aligned with the jet axis in HH 211. In addition, it has the same rotation sense as observed in $\mathrm{HH} 211$, with the blueshifted part in the SW and redshifted part in the NE (Lee09).

The observed field morphology is complicated, but may not be incompatible with the model, as it predicts different orientation depending on the region/distance. The NW field lines can trace the toroidal fields in the flattened envelope generated by the rotation. The SE field lines are further away from the center and thus can trace the fields at the outer edge of the flattened envelope, where the fields are still mainly poloidal. The SW field lines are slightly pinched and thus can trace the hourglass field lines dragged in by the gravitational collapse. The NE field lines are located closer to the source, where the hourglass field lines are expected to be dragged in more by the gravitational collapse and thus bent to be roughly perpendicular to the jet axis (see also Fig 4a in Allen, Li, \& Shu 2003). Note that it is also possible that the field lines there trace the toroidal fields generated by the rotation, as claimed in the case of NGC 1333 IRAS 4A where field lines were also detected perpendicular to the jet axis (Crutcher 2012). In addition, the NE/SW asymmetry could also be partly due 
to the presence of a secondary source. The polarization is not detected in all parts of the envelope, probably because of depolarization due to the complicated field morphology and our insufficient angular resolution (Kataoka et al. 2012). Further observations at higher sensitivity and resolution are needed to confirm the different field orientations in different regions.

Hourglass field morphologies have been seen in other Class 0 systems, e.g., NGC 1333 IRAS 4A (a binary system) at $\sim 400 \mathrm{AU}$ resolution (Girart et al. 2006) and $\mathrm{L}$ 1157 at 300-525 AU resolution (Stephens et al. 2013). At higher, $\sim 180 \mathrm{AU}$, resolution, toroidal field lines have also been detected tentatively in NGC 1333 IRAS 4A within $300 \mathrm{AU}$ of the center (Crutcher 2012). In HH 211, a partial hourglass field morphology has also been seen at $~$ 1000 AU resolution, with a pinched field morphology in the SW (ull et al. 2013). Here at higher, $\sim 170$ AU, resolution, we may have detected not only the hourglass field morphology in the SW further in but also the toroidal field lines in the flattened envelope within $300 \mathrm{AU}$ of the center. Thus, higher resolution observations are needed to reveal the toroidal fields, likely because the rotation dominates only in the very inner region in the Class 0 phase.

In current theory, the pinched geometry of the magnetic field in the flattened envelope can produce a magnetic braking, preventing a RSD to be formed at the center (Allen, Li, \& Shu 2003). However, such a RSD must have formed in order to launch the jet, as claimed in Lee09. It has been argued that a magnetic-field-rotation misalignment in a larger size scale could help the formation of the disk (Joos et al. 2012; Li et al. 2013). In the larger size scale in the cloud scale (arc-minute scale) (Matthews et al. 2009), the magnetic field lines are found to be roughly north-south oriented, neither aligned with nor perpendicular to the jet axis. However, the rotation axis in this scale is found to be roughly aligned with the jet axis (Lee09; Tanner \& Arce 2011). Thus, there is a misalignment of $\sim 30^{\circ}$ between the large-scale field axis and the rotation axis, and it may help the disk formation.

\section{JET}

Figure 2 shows the blueshifted (black contours) and redshifted (gray contours) components of the jet in $\mathrm{SiO}$, rotated clockwise to be aligned with the $\mathrm{x}$-axis. As seen in Lee09, the jet is highly collimated and consists of a chain of knotty shocks.

\subsection{Line Polarization and Field Morphology}

The figure also shows the polarization intensity map and vectors in the jet in the $\mathrm{SiO}$ line. The angles of the polarization vectors have an uncertainty of $\sim 13^{\circ}$. The line polarization can be attributed to the GK effect. In $\mathrm{HH}$ 211 , the jet lies close to the plane of the sky. For the $\mathrm{SiO}$ $\mathrm{J}=8-7$ emission, the optical depth is close to 1 and the radiative transition rate is greater than the collision rate in most regions (Lee09). All these are optimal for polarization detection and thus the polarization degree could exceed $10 \%$ (Kvlafis 1983), as seen in the observation. Two polarization detections, one in knot RK2 and one in between knots BK2 and BK3, are greater than $3 \sigma$, and thus should be real. Their polarization vectors have different orientations, with the former parallel to the jet axis and the latter inclined by $\sim 50^{\circ}$ to the jet axis. Other detections are below $3 \sigma$ and thus should be considered as tentative. No polarization is detected toward the bright innermost pair of knots probably because of the higher density there than other regions.

According to the GK effect, the field could be either parallel or perpendicular to the polarization vector, depending on the angle $\delta$ between the velocity flow axis and the polarization vector (Kvlafis 1983). If $35^{\circ} .3<\delta<54.7$, the field direction is parallel to the polarization vector. If $0<\delta<35^{\circ}$. 3 , then there is a $90^{\circ}$ ambiguity in the field direction. In the jet, the velocity flow axis is the same as the jet axis. The polarization vectors in between knots BK2 and BK3 have $\delta \sim 50^{\circ}$, thus the field lines there are parallel to the polarization vectors, highly inclined to the jet axis, and thus could be helical. In knot RK2, the polarization vectors are almost aligned with the velocity flow axis. In this case, the field lines could be either parallel or perpendicular to the polarization vectors. Therefore, the fields there could be either poloidal or toroidal.

\subsection{Discussion}

In knot RK2, there are sufficient number of polarization vectors for us to estimate the magnetic field strength there. According to Chandrasekhar \& Fermi (1953) and Ostriker et al. (2001), we can estimate the field strength in the plane of the sky with the following formula

$$
B \sim 0.5 \sqrt{4 \pi \rho} \frac{\triangle v_{\text {los }}}{\triangle \phi}
$$

The mass density $\rho=1.4 n_{\mathrm{H}_{2}} m_{\mathrm{H}_{2}} \sim 4.64 \times 10^{-16} \mathrm{~g} \mathrm{~cm}^{-3}$ with $n_{\mathrm{H}_{2}} \sim 10^{7} \mathrm{~cm}^{-3}$ (Lee09). The velocity dispersion along the line of sight $\Delta v_{\text {los }} \sim 10 \mathrm{~km} \mathrm{~s}^{-1}$ (Lee09). With the dispersion of the polarization angle $\triangle \phi \sim 20^{\circ} \pm 13^{\circ}$ (including the uncertainty in the angle of the polarization vectors), we have $B \sim 35_{-14}^{+65} \mathrm{mG}$. As discussed earlier, the field there could be toroidal. According to Shu et al. (1995), the toroidal field strength in an originally unshocked jet material could be $\gtrsim 7 \mathrm{mG}$ for the $\mathrm{HH} 211$ jet, which has a radius of $\lesssim 20$ AU (Lee09). Thus, if the field in knot RK2 is purely toroidal, our estimated field strength there is about 5 times as high, probably not unreasonable considering a shock compression. The magnetic pressure due to this toroidal field is $B^{2} / 8 \pi \sim 3 \times 10^{-5}$ dyne $\mathrm{cm}^{-2}$. The thermal pressure there is $1.2 n_{\mathrm{H}_{2}} k T \sim 8 \times 10^{-7}$ dyne $\mathrm{cm}^{-2}$ with $T \sim 500 \mathrm{~K}$ (Lee09), much lower than the magnetic pressure. Hence, the jet material there should be well confined by the magnetic field, if the field there is really toroidal.

Magnetic field morphology in protostellar jet is still poorly determined due to a lack of polarization detection. Previously, Carrasco-González et al. (2010) detected linear polarization in the synchrotron jet $\mathrm{HH} 80-81$ from the high-mass protostar IRAS 18162-2048 and found that the field lines there are mainly aligned with the jet axis. They saw an increase in polarization degree toward the jet edges and argued it to be due to helical field toward the jet edges. However, the jet with the polarization detection is far at $\sim 0.5 \mathrm{pc}$ away from the source and has an extremely large transverse width of $\sim 40000 \mathrm{AU}$, and thus might not trace the intrinsic jet coming from the source (Esquivel \& Raga 2013). In comparison, our polarization 
detections are within $2000 \mathrm{AU}\left(\sim 7^{\prime \prime}\right)$ of the source. The jet is located inside an outflow cavity and has a transverse width of $\lesssim 40 \mathrm{AU}$ (Lee09), as expected for a jet coming from a low-mass protostar. Since the polarization detections here show different orientations in different regions, further observations with ALMA at higher sensitivity are really needed to confirm them and to determine the field morphology.

In conclusion, we might have detected for the first time polarized $\mathrm{SiO}$ line emission in the jet due to the GK effect. If confirmed, our detection will open an perspective that
GK effect can be used to infer magnetic field morphology in the jet in the early phase of star formation, in which the jet is primarily molecular. The inferred field morphology can then be used to constrain the jet launching models.

We thank the anonymous referee for insightful comments. We thank the SMA staff for their efforts in running and maintaining the array. C.-F. Lee acknowledges grants from the National Science Council of Taiwan (NSC 1012119-M-001-002-MY3) and the Academia Sinica (Career Development Award).

\section{REFERENCES}

Allen, A., Li, Z., \& Shu, F. H. 2003, ApJ, 599, 363

Carrasco-González, C., Rodríguez, L. F., Anglada, G., et al. 2010, Science, 330, 1209

Chandrasekhar, S., \& Fermi, E. 1953, ApJ, 118, 113

Crutcher, R. M. 2012, ARA\&A, 50, 29

Esquivel, A., \& Raga, A. C. 2013, ApJ, 779, 111

Hirano, N., Ho, P. P. T., Liu, S.-Y., et al. 2010, ApJ, 717, 58

Girart, J. M., Rao, R., \& Marrone, D. P. 2006, Science, 313, 812

Girart, J. M., Crutcher, R. M., \& Rao, R. 1999, ApJ, 525, L109

Goldreich, P., \& Kylafis, N. D. 1982, ApJ, 253, 606

Goldreich, P., \& Kylafis, N. D. 1981, ApJ, 243, L75

Gueth, F. \& Guilloteau, S. 1999, A\&A, 343, 571

Ho, P. T. P., Moran, J. M., \& Lo, K. Y. 2004, ApJ, 616, L1

Hull, C. L. H., Plambeck, R. L., Bolatto, A. D., et al. 2013, ApJ, 768,159

Hull, C. L. H., Plambeck, R. L., Kwon, W., et al. 2014, ApJS, 213, 13

Joos, M., Hennebelle, P., \& Ciardi, A. 2012, A\&A, 543, A128

Kataoka, A., Machida, M. N., \& Tomisaka, K. 2012, ApJ, 761, 40

Kylafis, N. D. 1983, ApJ, 267, 137

Lai, S.-P., Girart, J. M., \& Crutcher, R. M. 2003, ApJ, 598, 392 ApJ, 639,292
Lee, C.-F., Ho, P. T. P., Palau, A., Hirano, N., Bourke, T. L., Shang, H., \& Zhang, Q. 2007, ApJ, 670, 1188

Lee, C.-F., Hirano, N., Palau, A., Ho, P. T. P., Bourke, T. L., Zhang, Q., \& Shang, H. 2009, ApJ, 699, 1584

Li, Z.-Y., Krasnopolsky, R., \& Shang, H. 2013, ApJ, 774, 82

Matthews, B. C., McPhee, C. A., Fissel, L. M., \& Curran, R. L. 2009, ApJS, 182, 143

Ostriker, E. C., Stone, J. M., \& Gammie, C. F. 2001, ApJ, 546, 980

Pudritz, R. E., Ouyed, R., Fendt, C., \& Brandenburg, A. 2007, Protostars and Planets V, 277

Rao, R., Girart, J. M., Lai, S.-P., \& Marrone, D. P. 2014, ApJ, 780, L6

Shu, F. H., Najita, J. , Ostriker, E. C. \& Shang, H. 1995, ApJ, 455, L155

Shu, F.H., Najita, J., Shang, H., \& Li, Z. -Y. 2000, in Protostars and Planets IV, ed. V. Mannings, A. P. Boss \& S. S. Russell (Tucson: University of Arizona Press), 789

Stephens, I. W., Looney, L. W., Kwon, W., et al. 2013, ApJ, 769, L15

Tanner, J. D., \& Arce, H. G. 2011, ApJ, 726, 40
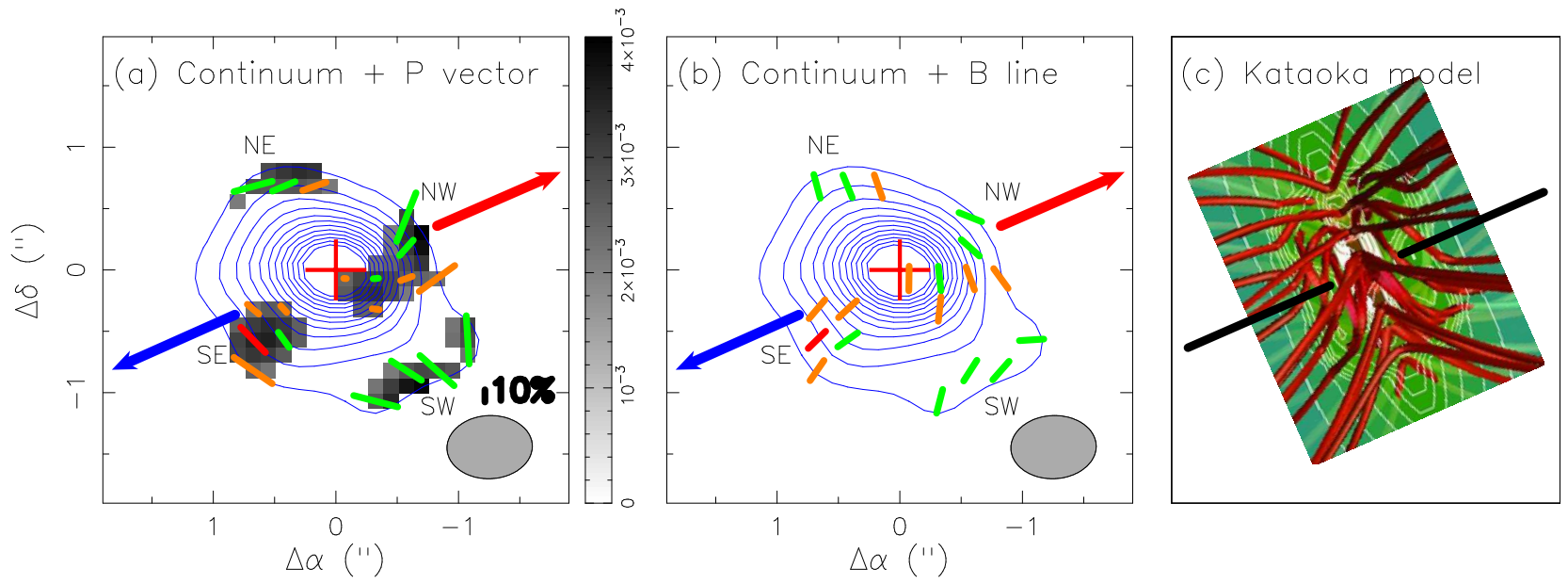

FIG. 1. - Continuum intensity, polarization intensity and vectors, field orientation, and model field morphology. In (a) and (b), the blue contours show the continuum intensity map. The rms noise level is $\sigma \sim 1.2 \mathrm{mJy}$ beam $^{-1}$. The peak has a flux density of $112 \mathrm{mJy}$ beam ${ }^{-1}$. The contours start from $5 \sigma$ with a step of $6 \sigma$. The cross marks the source position. The blue and red arrows show the approaching and receding sides of the mean jet axis, respectively. In (a), the gray-scale image shows the polarization intensity greater than $2 \sigma$ detection, where the rms noise level $\sigma=1.15 \mathrm{mJy}$ beam ${ }^{-1}$. Line segments show the polarization vectors for the detection with 2 to $3.5 \sigma$. (orange for $2-2.5 \sigma$, green for 2.5-3 $\sigma$, and red for 3-3.5 $\sigma$ ). Polarization degree is indicated by the length of the vector. In (b) Line segments show the magnetic field orientations. (c) shows the model density (white contours and color image) and field (red lines) morphology adopted from Kataoka et al. (2012), with the field axis (black lines) aligned with the jet axis of HH 211. 


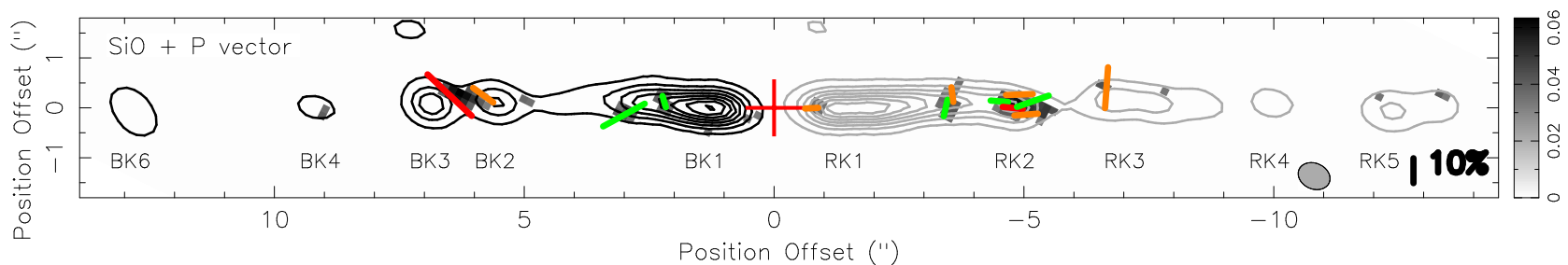

FIG. 2.- $\mathrm{HH} 211$ jet in $\mathrm{SiO} \mathrm{J}=8-7$, rotated clockwise to be aligned with the $\mathrm{x}$-axis. The black and gray contours show the blueshifted and redshifted components of the jet, respectively. The contours start from $4 \sigma$ with a step of $9 \sigma$, where $\sigma \sim 20 \mathrm{mJy}^{\mathrm{b}} \mathrm{beam}^{-1}$. The systemic velocity in HH 211 is $9.2 \pm 0.3 \mathrm{~km} \mathrm{~s}^{-1}$ LSR (Lee09). The blueshifted component is obtained by averaging the emission over the blueshifted velocity from -14 to $2 \mathrm{~km} \mathrm{~s}^{-1}$ and the redshifted component over the redshifted velocity from 19 to $37 \mathrm{~km} \mathrm{~s}^{-1}$. The gray-scale image shows the line polarization intensity greater than $2 \sigma$ detection, where the rms noise level $\sigma=18 \mathrm{mJy} \mathrm{beam}^{-1}$. The color and length of the polarization vectors have the same meanings as those in Figure 1 